\title{
Zinc supplementation in pre-diabetes: study protocol for a randomized controlled trial
}

\author{
Priyanga Ranasinghe ${ }^{1 *}$, Ranil Jayawardena ${ }^{2}$, ASAD Pigera ${ }^{1}$, Prasad Katulanda ${ }^{3}$, Godwin R Constantine ${ }^{3}$ \\ and Priyadarshani Galappaththy ${ }^{1}$
}

\begin{abstract}
Background: The number of people with diabetes is increasing worldwide, especially in developing South Asian countries. Therefore, preventing diabetes at the early stages has become an important issue. Recent clinical trials, systematic reviews, and meta-analysis have shown that zinc has beneficial effects on glycemic and metabolic control in those with diabetes. The present study is designed to evaluate the effects of zinc supplementation on glycemic control and other metabolic parameters in those with pre-diabetes.
\end{abstract}

Methods/design: The study will be conducted as a randomized, double-blind, placebo-controlled clinical trial for a period of 12 months at the Faculty of Medicine, University of Colombo, Sri Lanka. The study has been approved by the Ethics Review Committee of Faculty of Medicine, University of Colombo (EC/11/189). A total of 200 adults (age 18-60 years) with pre-diabetes will be recruited for the study. They will be stratified according to age, gender, and body mass index and randomly assigned into the test and placebo groups on a 1:1 ratio. The zinc capsules, each weighing $456 \mathrm{mg}$, will contain the following ingredients:zinc sulfate monohydrate $55.096 \mathrm{mg}$ (elemental zinc 20 mg), lactose monohydrate $399.504 \mathrm{mg}$, and stearic acid $1.400 \mathrm{mg}$. The placebo capsule with the same weight will be comprised of lactose monohydrate $454.600 \mathrm{mg}$ and stearic acid $1.400 \mathrm{mg}$. The subjects will receive either zinc $20 \mathrm{mg}$ capsules or placebo daily for a period of 12 months. The study drugs will be double blinded to both investigators and subjects. The visits and the evaluations will be done as follows: screening (visit 0), 1 month (visit 1), 3 month (visit 2), 6 month (visit 3), and 12 month (visit 4). The following primary outcome measures will be evaluated:fasting plasma glucose (FPG), post oral glucose tolerance test (OGTT), serum insulin, HbA1c, total/lowdensity lipoprotein (LDL)/high-density lipoprotein (HDL) cholesterol, triglycerides, serum zinc, and appetite using a visual analog scale. Secondary outcome measures include: blood pressure, anthropometry, and dietary assessment using a validated food frequency questionnaire. Data will be analyzed using SPSS v16.

Discussion: The present protocol will aim to establish the beneficial effects of zinc supplementation on disease progression in those with pre-diabetes and also establish its effectiveness in the prevention of diabetes mellitus.

Trial registration: Sri Lanka Clinical Trial Registry: SLCTR/2012/010

Keywords: Zinc supplementation, Pre-diabetes, Sri Lanka, Adults

\footnotetext{
* Correspondence: priyanga.ranasinghe@gmail.com

${ }^{1}$ Department of Pharmacology, Faculty of Medicine, University of Colombo,

Colombo, Sri Lanka

Full list of author information is available at the end of the article
}

\section{Biomed Central}

(c) 2013 Ranasinghe et al.; licensee BioMed Central Ltd. This is an Open Access article distributed under the terms of the Creative Commons Attribution License (http://creativecommons.org/licenses/by/2.0), which permits unrestricted use distribution, and reproduction in any medium, provided the original work is properly cited. 


\section{Background}

The prevalence of diabetes and pre-diabetes is increasing worldwide because of population growth, aging, urbanization, and increasing prevalence of obesity and physical inactivity. The South Asian region is currently experiencing an epidemic of diabetes and its associated complications [1,2]. The projected increase in prevalence of diabetes is out of proportion to the estimated increase in population [3]. In 1990, the prevalence of diabetes in Sri Lanka was 2.5\%; however, most recent studies show more than 1/5 of Sri Lankan adults are dysglycemic $[4,5]$. The prevalence of diabetes and prediabetes in the Sri Lankan adult population in 2005-2006 was $10.3 \%$ and $11.5 \%$ respectively [5]. Studies from developed countries have shown that the direct cost involved in the treatment of diabetes and its associated complications are high [6]. Furthermore the indirect costs due to early mortality, morbidity, and loss of productivity are likely to be much higher. This is even more pronounced in developing countries of the South Asian region, which are faced with the highest burden of disease [1]. Therefore, simple and effective preventive measures for those at risk of developing diabetes are a timely requirement.

Insulin is stored in $\beta$-cells of the pancreas in granules that contains a variable number of zinc atoms. Studies have shown that zinc plays an important role in insulin synthesis, storage, secretion, and action, while also being involved in various stages of carbohydrate and protein metabolism $[7,8]$. Oxidative stress plays an important role in the pathogenesis of diabetes and its complications. Impaired synthesis of antioxidant enzymes, such as superoxide dismutase and glutathione peroxidase, may be associated with increased oxidative stress [9]. Zinc is an integral structural component of these antioxidant enzymes, and zinc deficiency may impair their synthesis. In-vitro studies in animal models and in humans have demonstrated that zinc supplementation improves glycemic control and other metabolic parameters in diabetes [10-13]. A recent systematic review and meta-analysis on the effects of zinc supplementation in patients with diabetes showed that zinc supplementation reduces blood glucose, total cholesterol, and LDL cholesterol while improving glycemic control as demonstrated by a reduction in HbA1c [14]. Zinc supplementation has also shown beneficial effects on insulin resistance and components of the metabolic syndrome in pre-pubertal obese children [15]. However, at present there are no studies evaluating the long-term effects of zinc supplementation in prevention of disease in those at risk of diabetes. The present study aims to evaluate the effects of zinc supplementation on the progression of disease in patients with pre-diabetes from Sri Lanka and determine the metabolic effects of zinc supplementation on glycemic control. In addition, we also aim to evaluate effect of zinc supplementation on body weight and appetite in those with pre-diabetes.

\section{Methods/design}

\section{Objectives and hypothesis}

Objectives: The study aims to evaluate the effects of zinc supplementation on the progression of disease in patients with pre-diabetes from Sri Lanka and determine the metabolic effects of zinc supplementation on glycemic control. Furthermore, we aim to evaluate the effects of zinc supplementation on appetite and body weight in patients with pre-diabetes.

Hypothesis: The glucose concentration of pre-diabetic patients who are treated with elemental zinc $20 \mathrm{mg}$ daily will be lower than that of the control group. We also hypothesize that other diabetes-related metabolic parameters (serum insulin, total cholesterol, triglyceride, HDL cholesterol, LDL cholesterol, and blood pressure) will be improved in the treatment group in comparison to the control group.

\section{Study design}

The study will be conducted as a randomized, doubleblind, placebo-controlled clinical trial for a period of 12 months to assess the efficacy of daily zinc $20 \mathrm{mg}$ supplementation in pre-diabetes. The study will be conducted at the Faculty of Medicine, University of Colombo, Sri Lanka. Figure 1 provides an overview of the study.

\section{Sample size}

The number of patients required for determination of a $20 \%$ reduction of fasting plasma glucose in the treatment arm in comparison with the placebo arm at $90 \%$ power and $95 \%$ confidence interval with a dropout rate of $30 \%$ is 100 patients per arm. Hence, a total of 200 subjects with pre-diabetes will be recruited for the study.

\section{Population}

Pre-diabetes is defined as the presence of fasting plasma glucose levels between $110-125 \mathrm{mg} / \mathrm{dl}$ or 2-hpost-oral glucose plasma glucose levels between 140-199 mg/dl or both [World Health Organisation (WHO) 2006 criteria] [16]. Study participants will be recruited for the study after obtaining informed written consent.

\section{Inclusion and exclusion criteria Inclusion criteria}

- Both genders between the ages of 18-60 years, eligible for study through a screening test confirming the presence of pre-diabetes as defined above.

\section{Exclusion criteria}

- On any other vitamin or mineral supplementations or the current use of a weight loss medicine or dietary modification. 


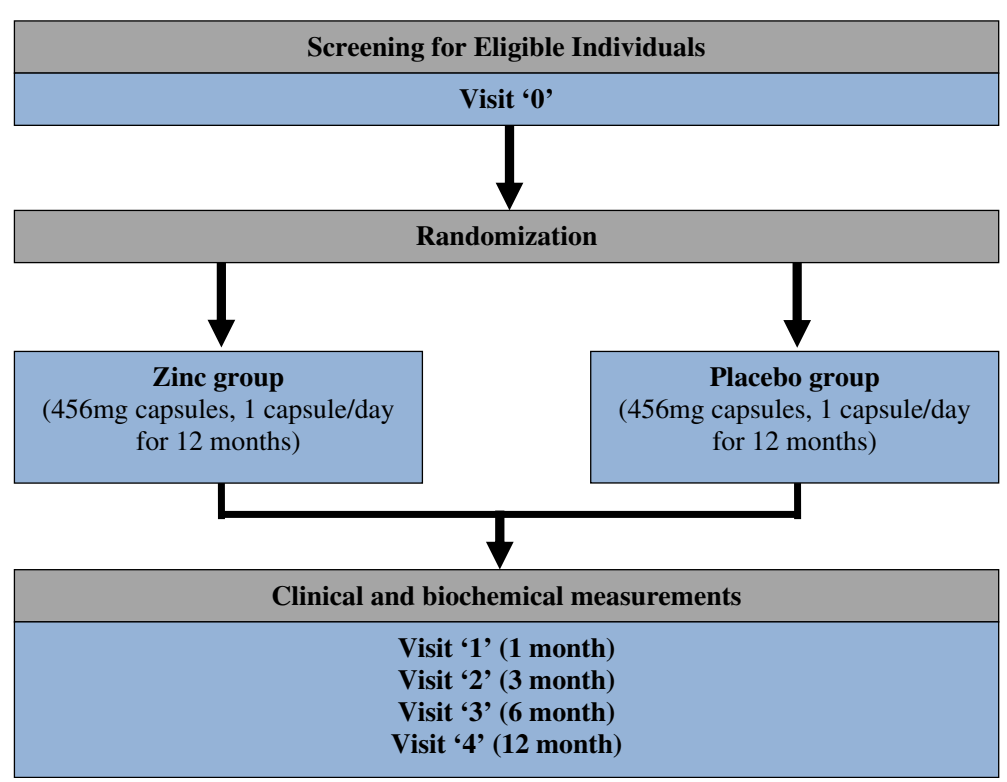

Figure 1 Schematic representation of study design.

- History of diabetes mellitus or any metabolic disease.

- Alcohol consumption > 20 g/day.

- Presently having acute diseases or other untreated illness requiring treatment.

- Impaired hepatic or renal functions.

- Lactation, pregnancy or unwillingness to use an effective form of birth control for women of childbearing years.

- History or presence of any condition, in the investigator's opinion, that would endanger the individual's safety or affect the study result.

\section{Suspension criteria}

- Subject's demand to discontinue the study.

- Serious adverse events or unusual changes in clinical test results.

- Principal investigator's decision to terminate the study (low rates of compliance, complications, or unable to sustain the study for various reasons).

\section{Randomization}

Subjects will be stratified according to age, gender, and body mass index and randomly assigned into the test and placebo groups at a 1:1 ratio according to the 'random number table' generated by SPSS v16.0 software package (SPSS Inc., Chicago, IL, USA).

\section{Blinding}

The study drugs are double blinded to both investigator and subject. The drug manufacturing will be done by SAP enterprises (PVT) Ltd., Colombo, Sri Lanka, and they will be responsible for the labeling of the zinc capsule and placebo with code numbers.

\section{Interventions}

The treatment drug is a capsule containing elemental zinc $20 \mathrm{mg}$ as the active ingredient; it will have a whitecolored body and a cap. The constituents of the zinc capsule are summarized in Table 1 . The placebo capsule will contain lactose monohydrate $(454.600 \mathrm{mg})$ and stearic acid $(1.400 \mathrm{mg})$. The placebo will be manufactured to have a similar appearance, shape, weight, taste, and color as thezinc $20 \mathrm{mg}$ capsule. The subjects will receive either one capsule of $20 \mathrm{mg}$ zinc or an identical placebo daily, taken $1 \mathrm{~h}$ before breakfast for a period of 12 months.

\section{Study groups}

a. Treatment group: zinc capsule

b. Control group: placebo

Table 1 Constituents of zinc capsule

\begin{tabular}{|c|c|c|c|}
\hline & Specification & $\begin{array}{l}\text { Quantity } \\
\text { per capsule }\end{array}$ & Status \\
\hline $\begin{array}{l}\text { Zinc sulfate }\left(\mathrm{ZnSO}_{4}\right) \\
\text { monohydrate* }\end{array}$ & USP & $55.096 \mathrm{mg}$ & $\begin{array}{l}\text { Active } \\
\text { ingredient }\end{array}$ \\
\hline Lactose monohydrate & $\mathrm{BP}$ & $399.504 \mathrm{mg}$ & Filler \\
\hline Stearic acid & USP & $1.400 \mathrm{mg}$ & Lubricant \\
\hline Total weight & & $456.00 \mathrm{mg}$ & \\
\hline
\end{tabular}

*Contains $20 \mathrm{mg}$ of elemental zinc. 


\section{Study period}

The study will be conducted for a period of 12 months. The visits and the evaluations will be done as follows: screening (visit 0), 1 month (visit 1), 3 month (visit 2), 6 month (visit 3), and 12 month (visit 4).

\section{Outcomes}

Primary outcome index: The following biochemical assessments will be done at baseline, at the stated intervals, and on completion: FPG, OGTT, serum insulin, HbA1c, total cholesterol, triglycerides, LDL cholesterol, HDL cholesterol, and serum zinc. Furthermore, appetite will be evaluated using the visual analog scale (VAS).

Secondary outcome index:

a. Measurement of systolic (SBP) and diastolic blood pressure (DBP).

b. Anthropometric assessment such as body weight, height, body mass index (BMI), waist circumference (WC), hip circumference ( $\mathrm{HC})$, and waist: hip ratio (WHR).

c. Dietary assessment using a validated food frequency questionnaire (FFQ) [17].

The primary and secondary outcomes will be measured at the screening visit (visit 0), 1-month visit (visit 1), 3-month visit (visit 2), 6-month visit (visit 3), and 12-month visit (visit 4). The changes between visit 0 and visit 4 measurements will be analyzed for the primary outcome indexes.

Safety assessment index: The following information will be recorded/measured for the safety assessment: vital signs, general medical examinations, full blood count $(\mathrm{FBC})$, renal function test, liver function test, and adverse events. Liver profile, renal profile, and FBC will be done at the screening day (visit 0), after 6 months (visit 3), and at the end of the study (visit 4). All other assessments will be done at each of the four visits.

\section{Procedures}

\section{Recruitment}

Participants will be recruited by open advertisement in newspapers, online, and notice boards at hospitals. Those who see the trial poster will visit the trial site voluntarily.

\section{Study schedule}

The detailed items that will be measured at every visit are described in Table 2.

\section{Measurement tools}

\section{Anthropometric measurements}

Body weight will be measured using a calibrated electronic floor scale (SECA 815 by SECA GmbH \& Co. Kg., Hamburg, Germany) to the nearest $0.1 \mathrm{~kg}$. Height will be measured to the nearest $0.1 \mathrm{~cm}$ using an upright plastic portable Stadiometer (SECA 217 by SECA GmbH \&

Table 2 A brief study schedule at every visit

\begin{tabular}{|c|c|c|c|c|c|}
\hline & Visit 0 (screening visit) & Visit 1 (1 month) & Visit 2 (3 month) & Visit 3 (6 month) & Visit 4 (12 month) \\
\hline Informed consent form & $\cdot$ & & & & \\
\hline Recording demographic data & $\cdot$ & & & & \\
\hline Medical history taking & $\cdot$ & & & & \\
\hline Physical examination ${ }^{1}$ & . & $\cdot$ & $\cdot$ & $\cdot$ & $\cdot$ \\
\hline FPG & $\cdot$ & $\cdot$ & $\cdot$ & $\cdot$ & $\cdot$ \\
\hline OGTT & $\cdot$ & $\cdot$ & $\cdot$ & $\cdot$ & $\cdot$ \\
\hline Serum insulin & $\cdot$ & $\cdot$ & $\cdot$ & - & - \\
\hline Lipid profile ${ }^{2}$ & · & . & · & . & · \\
\hline $\mathrm{HbA1c}$ & $\cdot$ & $\cdot$ & $\cdot$ & $\cdot$ & $\cdot$ \\
\hline Serum zinc & $\cdot$ & $\cdot$ & $\cdot$ & $\cdot$ & $\cdot$ \\
\hline Liver function ${ }^{3}$ & $\cdot$ & & & $\cdot$ & - \\
\hline Renal function ${ }^{4}$ & $\cdot$ & & & · & $\cdot$ \\
\hline $\mathrm{FBC}^{5}$ & $\cdot$ & $\cdot$ & $\cdot$ & $\cdot$ & $\cdot$ \\
\hline Blood pressure & $\cdot$ & $\cdot$ & $\cdot$ & $\cdot$ & $\cdot$ \\
\hline Electrocardiogram & $\cdot$ & & & & $\cdot$ \\
\hline Food frequency questionnaire & $\cdot$ & $\cdot$ & $\cdot$ & $\cdot$ & $\cdot$ \\
\hline Appetite (VAS) & $\cdot$ & $\cdot$ & $\cdot$ & $\cdot$ & - \\
\hline
\end{tabular}

${ }^{1}$ Body weight, height, waist circumference and hip circumference; ${ }^{2}$ total cholesterol, triglyceride, LDL cholesterol, and HDL cholesterol; ${ }^{3}$ AST, ALT, g-GTP, total bilirubin, albumin, and total protein; ${ }^{4}$ creatinine and blood urea nitrogen (BUN); ${ }^{5} \mathrm{WBC}, \mathrm{RBC}$, hemoglobin (Hb), hematocrit, and platelet count. 
Co. Kg., Hamburg, Germany). BMI will be calculated as weight (in kilograms) divided by the square of height (in meters). Waist circumference (WC) will be measured with a non-elastic tape (SECA 203 by SECA GmbH \& Co. Kg., Hamburg, Germany) at a point midway between the lower border of the rib cage and the iliac crest at the end of normal expiration. Similarly, the hip circumference also will be measured at the widest part of the buttocks atthe intertrochanteric level to the nearest $0.1 \mathrm{~cm}$. All anthropometric measurements will be made by using standard equipment and following WHO guidelines.

\section{Dietary measurements}

A culturally validated FFQ will be used to obtain habitual intake of calorie, macro- and micronutrients [17]. A VAS, $10 \mathrm{~cm}$ in length with words anchored at each end, expressing the most positive and the most negative rating, will be used to assess hunger, satiety, fullness, and desire to eat.

\section{Compliance calculation}

Subjects are asked to return remaining drugs and their compliance will be evaluated by using the formula given below:

$$
\begin{aligned}
\text { Compliance }(\%)= & {\left[\frac{(\text { distributed drugs }- \text { remaining drugs })}{\text { distributed drugs }}\right] } \\
& \times 100
\end{aligned}
$$

\section{Statistical analysis}

Parametric and nonparametric statistical tests will be applied using SPSS version 16 (SPSS Inc., Chicago, IL, USA) and Stata/SE 10.0 (StataCorp., College Station, TX, USA) for the data analysis. For each of the outcomes, multilevel regression analysis will be used to examine differences between trial arms. For binary outcomes the model will be logistic and for continuous outcomes the model will be linear regression. All analyses will follow intention-to-treat principles and a pre-specified analysis plan. Where appropriate, sensitivity analyses will be conducted (for example, control for additional covariates and bootstrapped $\mathrm{p}$ values for skewed outcomes). In the case of missing data values, we will apply mean imputation and regression imputation where rates are low and consider multiple imputations where they exceed $10 \%$.

\section{Adverse effect evaluation}

The Recommended Daily Allowance (RDA) of zinc is 11 $\mathrm{mg}$ in adult males and $8 \mathrm{mg}$ in females [18]. In addition, it has been shown that the therapeutic window for zinc is between $9-40 \mathrm{mg}$. Hence, $20 \mathrm{mg}$ of elemental zinc is a safe dose for humans. However, in the event of a probable adverse reaction, the following precautions would ensure timely identification and management of patients:

- Reporting mechanisms will be put in place to ensure direct reporting of probable adverse events to the investigator by patients (via telephone, which will be available $24 \mathrm{~h}$ on all days).

- During follow-up visits, probable adverse events will be noted by history and examination and investigated in detail. All adverse effects observed will be documented in the case record form (CRF) (Additional file 1).

- All serious adverse events will be reported to the Ethics Review Committee, Faculty of Medicine, University of Colombo, and the National Pharmacovigilance Unit of the Department of Pharmacology, Faculty of Medicine, University of Colombo.

- A Data Safety Monitoring Board (DSMB) identified by the investigators will evaluate all the adverse events at regular intervals

- Investigations including liver function, renal function, and FBC will be assessed as detailed above.

- Termination of study: In the event of major adverse effects occurring in a significant proportion of the study population, the study would be terminated pending further investigation.

\section{Data collection}

Data collection will be performed according to the standard operating procedures by medically trained research assistants (RAs).

\section{Data and biological sample handling}

Data will be entered by a minimum number of dedicated staff and saved in a dedicated computer with password protection. Blood samples will be stored in a secure facility with redundant measures to ensure specimens are kept in compliant conditions at all times when in storage. Storage technologies with the capability of monitoring the temperature of samples around the clock will be utilized. After each analysis has been completed and with the approval of the principal investigator, the samples stored in the storage facility may be disposed of by the sample custodian. Asample disposal sheet (SDS) will be completed and kept for further reference.

\section{Ethical approvals}

The study has been approved by the Ethics Review Committee (ERC) of the Faculty of Medicine, University of Colombo (EC/11/189). The trial is also registered at the Sri Lanka Clinical Trials Registry (SLCTR/2012/010). The study will be conducted in compliance with the 
Declaration of Helsinki and the Good Clinical Practice (GCP) guidelines.

\section{Discussion}

In this article, we present a clinical trial design to evaluate the effects of zinc supplementation in those with pre-diabetes. To our knowledge this is one of the first randomized controlled trials evaluating the effects of long-term zinc supplementation in pre-diabetes. This study will provide the necessary groundwork for future large-scale multicenter clinical trials. Given the current enthusiasm for using various dietary supplements to improve glycemic control and metabolic parameters in those with dysglycemia, properly designed scientific evaluations are a timely requirement. Furthermore, in animal models and in humans, zinc plays an important role in the regulation of appetite [19]. However, presently there are no well-designed randomized control trials to support/refute this argument. This study will also help to provide information pertaining to appetite changes resulting from zinc supplementation. Appetite regulation could be one of the probable mechanisms responsible for beneficial effects on glycemic control. The result, positive or negative, should provide a step change in the evidence guiding current and future policies regarding dietary supplementation in prevention of diabetes.

\section{Trial status}

Patient recruitment stage.

\section{Additional file}

Additional file 1: Case Record Form (CRF).

\section{Abbreviations}

ALT: Alanine aminotransferase; AST: Aspartate aminotransferase; BMI: Body mass index; BUN: Blood urea nitrogen; CRF: Case report form; DBP: Diastolic blood pressure; DSMB: Drug safety monitoring board; ERC: Ethics review committee; FBC: Full blood count; FFQ: Food frequency questionnaire; FPG: Fasting plasma glucose; g-GTP: Gamma-glutamyltransferase; HC: Hip circumference; HDL: High-density lipoprotein; LDL: Low-density lipoprotein; OGTT: Oral glucose tolerance test; RA: Research assistance; RBC: Red blood cell; RDA: Recommended Daily Allowance; SBP: Systolic blood pressure; SDS: Sample disposal sheet; SLCTR: Sri Lanka clinical trial registry; SOP: Standard operating procedure; AS: Visual analog scale; WBC: White blood cell; WC: Waist circumference; WHO: World Health Organization; WHR: Waist: hip ratio.

\section{Competing interest}

The authors declare that they have no competing interests.

\section{Authors' contributions}

$P R$ and RJ substantially contributed to the general idea and design of the study. PR, RJ, PG, ASADP, PK, and GRC took part in designing the protocol. $P R, R J$, and PG planned the data analysis. PR and ASAD drafted the manuscript. All authors have read and consented to the manuscript.

\section{Acknowledgements}

This work is supported by a grant from the National Science Foundation (NSF) of Sri Lanka (RG/2012/HS/01).

\section{Author details}

'Department of Pharmacology, Faculty of Medicine, University of Colombo, Colombo, Sri Lanka. ${ }^{2}$ Institute of Health and Biomedical Innovation, Queensland University of Technology, Brisbane, QLD, Australia. ${ }^{3}$ Department of Clinical Medicine, Faculty of Medicine, University of Colombo, Colombo, Sri Lanka.

Received: 20 November 2012 Accepted: 4 February 2013

Published: 19 February 2013

\section{References}

1. Jayawardena R, Ranasinghe P, Byrne NM, Soares MJ, Katulanda P, Hills AP: Prevalence and trends of the diabetes epidemic in South Asia: a systematic review and meta-analysis. BMC Public Health 2012, 12:380.

2. Katulanda P, Ranasinghe $P$, Jayawardena $R$, Constantine GR, Sheriff $M H$, Matthews DR: The prevalence, patterns and predictors of diabetic peripheral neuropathy in a developing country. DiabetolMetabSyndr 2012, 4(1):21.

3. Wild S, Roglic G, Green A, Sicree R, King H: Global prevalence of diabetes: estimates for the year 2000 and projections for 2030. Diabetes Care 2004, 27(5):1047-1053.

4. Katulanda P, Sheriff MH, Matthews DR: The diabetes epidemic in Sri Lankaa growing problem. Ceylon Med J 2006, 51(1):26-28.

5. Katulanda P, Constantine GR, Mahesh JG, Sheriff R, Seneviratne RD, Wijeratne S, Wijesuriya M, McCarthy Ml, Adler Al, Matthews DR: Prevalence and projections of diabetes and pre-diabetes in adults in Sri Lanka-Sri Lanka Diabetes, Cardiovascular Study (SLDCS). Diabet Med 2008, 25(9):1062-1069.

6. Brandle M, Zhou H, Smith BR, Marriott D, Burke R, Tabaei BP, Brown MB, Herman WH: The direct medical cost of type 2 diabetes. Diabetes Care 2003, 26(8):2300-2304.

7. Zalewski PD, Millard SH, Forbes IJ, Kapaniris O, Slavotinek A, Betts WH, Ward $A D$, Lincoln SF, Mahadevan I: Video image analysis of labile zinc in viable pancreatic islet cells using a specific fluorescent probe for zinc. J HistochemCytochem 1994, 42(7):877-884.

8. Chausmer AB: Zinc, insulin and diabetes. J Am CollNutr 1998, 17(2):109-115.

9. Kelly F: Use of antioxidants in the prevention and treatment of disease. J Int Fed ClinChem 1998, 10(1):21-23.

10. Simon SF, Taylor CG: Dietary zinc supplementation attenuates hyperglycemia in db/db mice. ExpBiol Med (Maywood) 2001, 226(1):43-51.

11. Oh HM, Yoon JS: Glycemic control of type 2 diabetic patients after shortterm zinc supplementation. Nutr Res Pract 2008, 2(4):283-288.

12. Al-Maroof RA, Al-Sharbatti SS: Serum zinc levels in diabetic patients and effect of zinc supplementation on glycemic control of type 2 diabetics. Saudi Med J 2006, 27(3):344-350.

13. Gunasekara P, Hettiarachchi M, Liyanage C, Lekamwasam S: Effects of zinc and multimineral vitamin supplementation on glycemic and lipid control in adult diabetes. Diabetes MetabSyndrObes 2011, 4:53-60.

14. Jayawardena R, Ranasinghe P, Galappatthy P, Malkanthi R, Constantine G, Katulanda P: Effects of zinc supplementation on diabetes mellitus: a systematic review and meta-analysis. DiabetolMetabSyndr 2012, 4(1):13.

15. Hashemipour M, Kelishadi R, Shapouri J, Sarrafzadegan N, Amini M, Tavakoli N, Movahedian-Attar A, Mirmoghtadaee P, Poursafa P: Effect of zinc supplementation on insulin resistance and components of the metabolic syndrome in prepubertal obese children. Hormones (Athens) 2009, 8(4):279-285.

16. World Health Organization, International Diabetes Federation: Definition and diagnosis of diabetes mellitus and intermediate hyperglycemia: report of a WHO/IDF consultation. Geneva: World Health Organization; 2006.

17. Jayawardena R, Swaminathan S, Byrne NM, Soares MJ, Katulanda P, Hills AP: Development of a food frequency questionnaire for Sri Lankan adults. Nutr J 2012, 11(1):63.

18. Institute of Medicine Food and Nutrition Board: Dietary Reference Intakes for Vitamin A, Vitamin K, Arsenic, Boron, Chromium, Copper, lodine, Iron, Manganese, Molybdenum, Nickel, Silicon, Vanadium, and Zincexternal link icon. Washington DC: Institute of Medicine Food and Nutrition Board; 2001.

19. Mantzoros CS, Prasad AS, Beck FW, Grabowski S, Kaplan J, Adair C, Brewer $\mathrm{GJ}$ : Zinc may regulate serum leptin concentrations in humans. J Am Coll Nutr 1998, 17(3):270-275.

doi:10.1186/1745-6215-14-52

Cite this article as: Ranasinghe et al:: Zinc supplementation in prediabetes: study protocol for a randomized controlled trial. Trials 2013 14:52. 